\title{
ASO Author Reflections: How We Convey Empathy, Address Uncertainty, and Share Serious News: Challenges to Remote Surgical Care
}

\author{
Claire Sokas, MD, MPH (1), Christina Minami, MD, MS, and Quoc-Dien Trinh, MD \\ Department of Surgery, Center for Surgery and Public Health, Brigham and Woman's Hospital, Boston, MA
}

\section{PAST}

The coronavirus disease 2019 (COVID-19) pandemic laid bare susceptibilities in our healthcare system, halting the routine provision of primary and preventative services, taxing personal protective equipment supply chains, and highlighting disparities in access to care. Responding to the surge of acutely ill and potentially infectious patients forced hospitals to triage and delay care, including surgical cancer care. Although the significance of clinical delays to cancer surgery will take years to fully appreciate, we wanted to understand the individual experience of patients whose surgeries were delayed. We interviewed patients with early- and intermediate-stage breast and prostate cancers, who received the unexpected news of surgery cancellation in early May via phone calls in the midst of a case surge in Boston, MA. We found that, while many patients accepted these delays due to the unprecedented nature of a global pandemic, cancer- and COVID-related distress was prevalent. 'Our patients placed significant trust in their surgeons to guide them through these uncertain times.

\section{PRESENT}

A year later, case numbers and deaths are declining, and vaccination rates continue to rise. As we adjust to a "new normal," it is the ideal time to reflect on what changes to

(C) Society of Surgical Oncology 2021

First Received: 24 May 2021

Accepted: 25 May 2021;

Published Online: 23 June 2021

C. Sokas, MD, MPH

e-mail: csokas@bwh.harvard.edu healthcare may be permanent. Prior to the pandemic, utilization of telehealth in cancer care was limited and variable, with notable barriers including access to technology and inconsistent reimbursement. ${ }^{2}$ Significant strides have been made to expand telehealth services, both through improved access to technology and more consistent insurance reimbursement. ${ }^{3,4}$ The pandemic has served as a realworld trial of implementation of telehealth services, and while success is not guaranteed, telehealth holds a clear advantage when considering expanded access to care, both in terms of geographic and social distance.

\section{FUTURE}

Both patients and physicians have expressed concern regarding the use of telehealth for sensitive conversations, such as a new cancer diagnosis or complex treatment plan. ${ }^{1,5}$ The successful implementation of telehealth tailored to cancer care mandates a new approach to teaching and practicing empathetic communication and establishing the patient-physician bond. There is still a need for best practice recommendations regarding which perioperative visits can be feasibly and successfully completed via telehealth. Many communication tools, such as SPIKES (setting, perception, invitation, knowledge, empathy/emotion and strategy/summarize), have been adapted to address specific telehealth needs, including minimizing distractions, addressing virtual privacy, and ensuring appropriate technical setup. ${ }^{5}$

Next, surgeons should anticipate a higher prevalence of distress for patients with a cancer diagnosis during this time and evaluate for distress early and frequently. Distress screening, through single or multi-item questionnaires, needs to be adapted to a telehealth platform; options include making questionnaires available via an electronic health portal prior to the visit, or having a member of the 
healthcare team screen patients at each visit. A positive screen for distress should trigger appropriate referrals for social support (social work, support groups) in real time and a communication hand-off from surgeon to such services.

Finally, surgeons must be judicious in the use of telehealth for vulnerable populations. Internet access is not universal, nor is access to broadband services that make telehealth possible. ${ }^{4}$ Older adults, as well as patients on public insurance or living below the poverty line, are less likely to regularly use the internet in general or telehealth specifically. ${ }^{4,6}$ Changes in service lines to expand telehealth must ensure equity in access to care for all patients, particularly those whose access to care is already hindered.

\section{CONCLUSION}

The pandemic has challenged patients and physicians to rapidly adapt to new care models. Telehealth has become essential to ongoing cancer care, and by utilizing existing communication tools and proactively addressing patient distress, surgeons can continue to establish trusting relationships with their patients regardless of the distance. However, the expanded use of telehealth must be structured to accommodate the needs of all patients and preserve equity of accessing care.
DISCLOSURES Quoc-Dien Trinh: Astellas, Bayer, Janssen (consultant), Intuitive Surgical (grant).

\section{REFERENCES}

1. Sokas CM, Kelly M, Sheu C, Song J, Welch HG, Bergmark R, Minami C, Trinh QD. Cancer in the shadow of COVID: earlystage breast and prostate cancer patient perspectives on surgical delays due to COVID-19. Ann Surg Oncol. 2021. https://doi.org/ 10.1245/s10434-021-10319-0.

2. Heyer A, Granberg RE, Rising KL, Binder AF, Gentsch AT, Handley NR. Medical oncology professionals' perceptions of telehealth video visits. JAMA Netw Open. 2021;4(1):e2033967.

3. Centers for Medicare and Medicaid Services. List of telehealth services. https://www.cms.gov/Medicare/Medicare-General-Infor mation/Telehealth/Telehealth-Codes. April 9, 2021. Accessed 16 May 2021.

4. Patel SY, Mehrotra A, Huskamp HA, Uscher-Pines L, Ganguli I, Barnett ML. Variation in telemedicine use and outpatient care during the COVID-19 pandemic in the United States: study examines variation in total US outpatient visits and telemedicine use across patient demographics, specialties, and conditions during the COVID-19 pandemic. Health Affairs. 2021;40(2):349-58.

5. Holstead RG, Robinson AG. Discussing serious news remotely: navigating difficult conversations during a pandemic. JCO Oncol Pract. 2020;16(7):363-8.

6. Perrin A, Atske S. 7\% of Americans don't use the internet: who are they? Report. Pew Research Center. April 2, 2021. Accessed 14 May 2021.

Publisher's Note Springer Nature remains neutral with regard to jurisdictional claims in published maps and institutional affiliations. 